\title{
INVESTIGACIONES
}

\section{Investigación-acción y método de proyectos en educación física: organización de un torneo de marro}

\author{
Investigation-action and the projects methodology in physical education: \\ The organization of Prisoner's Base tournament \\ A pesquisa-ação e método de projeto em educação física Proyecctos: \\ a organização de um torneio de barres
}

\author{
Jordi Brasó i Rius, ${ }^{a}$ Xavier Torrebadella Flix ${ }^{b}$ \\ anstituto del Centro de Alto Rendimiento Deportivo. San Cugat del Vallés, Barcelona \\ Telf.: 0034605360482. Correo electrónico: jbraso@ xtec.cat \\ bUniversidad Autónoma de Barcelona. Bellaterra, Barcelona \\ Telf.: 0034619291081. Correo electrónico: xtorreba@gmail.com
}

\begin{abstract}
RESUMEN
El artículo expone una experiencia educativa desde el área de educación física mediante la metodología de proyectos. La propuesta consistió en la organización de un torneo del juego popular del marro. Los organizadores fueron 32 alumnos de un Curso de Acceso al Grado Superior, de un Instituto público del Vallés Occidental, de la provincia de Barcelona. La experiencia se enmarca en la metodología de investigación-acción. La preparación del evento, conducido autónomamente por los propios alumnos, duró dos trimestres, en los cuales los escolares tuvieron que encargarse de todos los aspectos necesarios para la óptima realización del evento final. Los resultados obtenidos detallan que el proyecto representó un poderoso recurso para alcanzar los objetivos competenciales curriculares. A la vez, presentó un enfoque constructivista hacia propuestas didácticas de una pedagogía crítica, que sitúan al alumnado en un entorno escolar afectivo y que les concede espacios de autonomía para el desarrollo personal y académico.
\end{abstract}

Palabras clave: cesión de autonomía, educación física crítica, juego del marro, juegos tradicionales, proyecto educativo.

\section{ABSTRACT}

This article exposes an educative experience in the physical educational area by using the projects based methodology. The experience consists in the organization of the popular Prisoner's Base game. It was organized by 32 students in a prep course conducting to a Superior Grade (post-school studies that are only possible to do after getting "level A" or by passing this course) of a public school of Vallés Occidental, a Barcelona province. The experience is based in the action-research methodology. The event preparation, conducted autonomously by the students, lasted two quarters, in which they have to take care of all the necessary aspects for the optimal realization of the final event. The results obtained show that the project presents a powerful resource to achieve the competence-based curricular objectives. Besides, it presents a constructivist approach to didactic proposals of critic pedagogy, that locate the student in an affective scholar environment that grants them spaces of autonomy for the personal and academic development.

Key words: autonomy's assignment, critic physical education, prisoner's base game, traditional games, educative project. 
La rutina nos lleva a estar hoy como ayer, mañana como hoy y siempre igual (Blanco, 1906).

\section{INTRODUCCIÓN}

Los juegos en la educación de la infancia y de la juventud han sido, desde hace años, un recurso pedagógico muy valorado. Quintiliano de Calahorra, Luis Vives, Martín Sarmiento, Vicente Naharro o Pere Vergés han dejado una profunda huella histórica sobre las aplicaciones didácticas del juego (Bantulà, 2006; Dahlman, 2011; De Borja, 1984; Moro, 2007; Torrebadella, 2011).

Sin duda alguna, el juego motor es por excelencia el portador de la cultura lúdica en el marco escolar. Desde la institucionalización de la educación física, los juegos corporales han sido utilizados tanto para contribuir a la salud y al desarrollo de competencia motriz, como para contribuir a la educación moral y a la formación del carácter.

En la educación contemporánea, dicha institucionalización debemos situarla primeramente en la escuela de Salzmann, en Schnepfenthal, con la aportación de Johann Christoph Friedrich GutsMuths (1759-1839) y su obra Juegos para ejercicio y recreo del cuerpo y del espíritu dedicado a la juventud (1796); un compendio recopilatorio de juegos gimnásticos con finalidad higiénico-pedagógica y moralizadora.

La segunda gran aportación tiene como protagonista al reverendo Thomas Arnold y la labor institucional del deporte contemporáneo en la escuela de Rugby. En este lugar, Arnold emprende uno de los fenómenos sociales más trascendentes de la época contemporánea: "Hacer de la organización deportiva, puesta en manos de los escolares mismos y funcionando bajo su cuidado, la escuela práctica de la sociedad", una idea que para Valserra (1944: 249) se convertiría en la 'piedra angular del Imperio Británico', y cuya trascendencia será imitada y reconocida internacionalmente.

En la educación a través del deporte, Arnold puso en manos de los propios alumnos la responsabilidad -self-government- del desarrollo de las habilidades para la vida (Almeida, 2003; Vilanou y De Bolòs, 2005). De todas formas, en la actualidad, este modelo de intervención docente todavía busca su legitimización social y pedagógica (Cecchini, Montero y Peña, 2003).

Desde entonces, los juegos y el deporte, como la propia educación física, han sido portadores de vínculos de poder que han pugnado por "la apropiación de los usos sociales legítimos del cuerpo" con intereses para definir subjetividades y hegemonías sociales (Varela, 1991: 29). Sin embargo, actualmente los enfoques constructivistas de la educación física se sitúan en horizontes y disputas de contenidos y metodologías diversas que tratan de responder eficazmente a los problemas del entorno social. Las propuestas en una enseñanza basada en la cesión de autonomía al alumnado, se revelan ante la tradición escolástica de cuya herencia cuesta todavía separarnos.

Es en el marco de una pedagogía crítica, que la educación física puede responder a traspasar los umbrales de los heredados regímenes disciplinarios de la educación. Reconociendo el currículo oculto (Barbero, 1996; Kirk, 1990) y los mismos códigos disciplinares con los que la educación física queda atrapada en la construcción de los regímenes escolares (Vicente, 2012), las apuestas hacia una educación física crítica pueden 
presentarse como poderosos recursos didácticos hacia la transformación de las prácticas sociales (Gómez y Prat, 2009; López, 2012; López y Gea, 2010).

Los juegos tradicionales y deportivos en manos de los propios protagonistas se revalorizan como metodología no directiva en la cesión de autonomía y predisponen situaciones que favorecen la motivación, la iniciativa personal, la toma de decisiones, el autocontrol, las relaciones de cooperación, el esfuerzo compartido, el acuerdo democratizado en la concreción y aceptación de las normas, el juego limpio y el éxito colectivo; son, en definitiva, potenciadores de valores (re)creativos y formativos.

En este cambio de paradigma didáctico basado en la cesión de la autonomía están siendo reconocidas en la educación física desde propuestas que van desde la enseñanza primaria hasta la enseñanza superior (Martos et al., 2014; Moreno, Aracil y Reina, 2014; Vera, 2010). Es en estos últimos años que en España se han presentado algunos estudios experimentales (Belando, Ferriz-morell y Moreno-murcia, 2012; Moreno, Gómez y Cervelló, 2010; Moreno y Vera, 2008; Vera, 2010; Vera y Moreno, 2009) que constituyen un excelente marco teórico para iniciar un cambio de modelo metodológico en la enseñanza. No obstante, los reparos del profesorado (Vera y Moreno, 2007) son obstáculos que debemos franquear a partir de proponer intervenciones docentes prácticas que sirvan de modelos didácticos (Becerro y Torrebadella, 2013; Torrebadella, 2013).

Los juegos tradicionales tienen en la educación física un espacio revelador en cuanto a su dimensión de contextualización e interpretación histórica siempre susceptible de ser conducente hacia un enfoque de la pedagogía crítica (Becerro y Torrebadella, 2013; Camerino y Castañer, 1984; Devís, 2008; Devís y Peiró, 1992; Lavega, 2006; Navarro, 2011; Vicente y Brozas, 1996). Es, pues, a través de las palabras de David Kirk que también nos posicionamos para presentar la presente propuesta didáctica:

Si la educación física quiere verdaderamente servir a los intereses emancipatorios de la mayoría de los alumnos en las escuelas, nuestros esfuerzos y energías deben dirigirse hacia estos temas y no hacia los intereses del deporte de elites, la industria, el militarismo y los intereses de una sociedad represiva y conformista (Kirk, 1990: 167).

En este sentido, el siguiente trabajo presenta una experiencia educativa llevada a cabo en un centro escolar, en el ámbito la educación física. Se ha pretendido recuperar una de las muchas iniciativas que surgieron a inicios del s. XX en el movimiento conocido como la Escuela Nueva, y que significaron un alto grado de prestigio por toda Europa.

Concretamente, se trata una experiencia mediante un proyecto educativo que significó una novedad en la enseñanza a inicios del pasado siglo. Los proyectos educativos implicaban una forma de aprender mediante la autonomía, lo que suponía una alta motivación, además de abarcar temas transversales y no solamente de una única materia.

El punto de inicio del trabajo por proyectos basados en el método científico hay que buscarlo en John Dewey - y en su discípulo William Heard Kilpatrick- y la renovación pedagógica en Estados Unidos. Kilpatrick define un proyecto como "un acto o una serie de actos que el niño realiza voluntariamente con un fin dado, y esa realización se efectúa en un ambiente social bien definido, eso es, en la clase, que es una verdadera comunidad infantil" (Butts, 1930: 22). Y aunque la idea organizativa inicial del método no se pueda cumplir completamente en la actualidad, ya que por ejemplo se incidía en el mismo acto voluntario del trabajo o en la importancia de no emplear el tiempo de manera rígida, hay características 
que aun es posible mantener en esta manera de educar, entre ellas el trabajo en grupo, la resolución de problemas y autonomía, la iniciativa por parte del alumno, el papel consultivo y secundario del profesor, el contacto directo con la realidad para tratar los conceptos, la interdisciplinariedad de la propuesta, y el uso de una enseñanza globalizada. De este modo, se encuentran diversas propuestas que han optado por el método de proyectos (Angulo, Betanzo y López, 2005; Lacueva, Imbernón y Llobera, 2004; López y Lacueva, 2007; Martín, 2011; Mérida et al., 2011; Trobajo y Salvatella, 2003). Esta metodología de enseñanza, entre otros aspectos, potencia el interés, implica al alumnado y permite a la vez una globalización del currículum (Blanco, 1996; Butts, 1930; Ferrándiz, 2005; Martín, 2011; Pring, 1976; Rich, 2000; Rodríguez, 1995; Torres, 1987; Zabala, 1999). Es relevante además apuntar, como cita Ovidio Decroly, que estos proyectos provienen de un centro de interés (Rodríguez, 1995).

La iniciativa que se pretendió recuperar con esta experiencia es la que inició en 1922 el pedagogo Pere Vergés. En ella, mediante la vida social en la escuela, dentro de la cual se incluían los juegos deportivos competitivos, se pretendía formar a los alumnos, es decir, hacer de ellos ciudadanos. En los centros educativos liderados por el pedagogo, se establecían grupos, a modo de clubes, que competían en multitud de concursos, tanto deportivos como relacionados con la vida social, o también con el aspecto académico (Brasó y Torrebadella, 2014; González-Agàpito, 1998; Saladrigas, 1973; Vergés, 1932, 1947; Vilanou, 1998). La experiencia, por lo tanto, pretende ser una adaptación a este modelo lúdico-competitivo, pero además se ha utilizado para recuperar uno de los juegos populares que a inicios del s. XX practicaban los niños, y que actualmente ha quedado olvidado: el juego del marro. Este juego motriz de equipo tiene unas características por lo que respecta a su lógica interna que lo hacen mucho más complejo que los actuales deportes colectivos (Parlebas, 2001; Torrebadella y Brasó, 2014). Por lo tanto, además de hacer un trabajo por proyectos centrado en la cesión de autonomía al alumnado, con la propuesta también se ha recuperado una pequeña parte de la historia recién olvidada de los juegos.

\section{DESCRIPCIÓN DEL PROYECTO}

\subsection{CONTEXTUALIZACIÓN}

La propuesta se llevó a cabo en la población de San Cugat del Vallés (Barcelona), de ochenta y cinco mil habitantes. El centro donde se intervino fue el Instituto del Centro de Alto Rendimiento, donde realizan ciclos formativos de la familia de las actividades físico-deportivas, además de llevarse a cabo un curso de preparación para acceder al grado superior de Animación de actividades físico-deportivas, destinado a 35 alumnos. Este curso de acceso al Grado Superior (CAS) es accesible para los alumnos que han obtenido un ciclo formativo de grado medio, tiene una duración de un curso académico, y la nota final obtenida facilita el acceso al grado superior. Dentro del programa preparatorio se incluyen tres materias de modalidad: Catalán, Castellano e Inglés; y dos específicas: Matemáticas y Educación física.

Es en esta última materia, de dos horas semanales, donde se enmarca la propuesta en cuestión. Además trabajar los contenidos específicos marcados por el currículum de forma más secuenciada, también se propuso un trabajo por proyectos de tipo productivo (Rodríguez, 1995): la organización de un torneo de marro. 
La elaboración de la propuesta por parte del grupo-clase se fundamenta en una investigación previa (Brasó y Torrebadella, 2014; Torrebadella y Brasó, 2014) en la que se analiza de un lado la significatividad del juego, y del otro todo el potencial del método de proyectos y por competencias, ya actuales, pero sobre todo muy presentes en los inicios del s. XX con el movimiento de la Escuela Nueva. Se optó por tratar el juego del mismo modo como se había hecho en el ámbito escolar, concretamente en el modelo pedagógico de Pere Vergés en la Antigua Escuela del Mar de la Barceloneta (Barcelona), de 1922 a 1938 y en las colonias de Vilamar (Calafell), año 1922 y el período de 1930 a 1932 (GonzàlezAgàpito, 1998; Saladrigas, 1973; Vergés, 1932, 1957; Vilanou, 1998).

\subsection{UNIDADES DIDÁCTICAS, OBJETIVOS Y CONTENIDOS TRABAJADOS}

Para justificar el proyecto correspondiente en la programación del curso, es conveniente justificar todo el conjunto de unidades didácticas trabajadas. De este modo, teniendo en cuenta el Temario y criterios de evaluación del curso de formación específico para el acceso de ciclos formativos de grado superior, en la materia de Ciencias del Deporte ${ }^{1}$, se desarrollaron cinco unidades didácticas (Tabla 1).

Tabla 1. Duración de las unidades didácticas de la materia de Ciències de l'Esport

\begin{tabular}{|c|c|}
\hline Título de la Unidad didáctica & Duración \\
\hline 1. Sociología e historia de la actividad física y el deporte & $16 \mathrm{~h}$ \\
\hline 2. La actividad física y la salud & $12 \mathrm{~h}$ \\
\hline 3. Actividades físico-deportivas I & $16 \mathrm{~h}$ \\
\hline 4. Actividades físico-deportivas II & $20 \mathrm{~h}$ \\
\hline 5. Motricidad y aprendizaje motor & $9 \mathrm{~h}$ \\
\hline TOTAL (Curso escolar: 2h/semana) & $73 \mathrm{~h}$ \\
\hline
\end{tabular}

Estas cinco unidades didácticas pretenden dar cabida a todos los contenidos y criterios de evaluación correspondientes a los bloques que marca el currículum: Sociología e historia de la actividad física y el deporte, Motricidad y aprendizaje motor, Actividad física y salud y Actividades físico-deportivas.

El carácter transversal del proyecto requería que fuese tratado en la mayor parte de las unidades didácticas posibles. De este modo, desde el inicio de curso a finales de septiembre, hasta el día del evento a mediados de abril, se fue trabajando sobre la propuesta. Como consecuencia, el proyecto se desarrolló desde todas las unidades presentadas, excepto la

Temario consultable en el enlace (tomado el 10 de octubre de 2014):

http://www.xtec.cat/alfresco/d/d/workspace/SpacesStore/25850dd9-5182-4b79-834b-7d45bf2494f3/temari_cas_ciencies_ esport.pdf 
última relacionada con la motricidad y el aprendizaje motor. La justificación vino dada por ser dicha unidad didáctica la que se realizó posteriormente a la fecha del evento final y, por lo tanto, el proyecto se daba por concluido. Además, la voluntad durante el proceso era no limitarse solamente a la materia, sino que se pretendía tratar muchos otros contenidos transversales (Ferrándiz, 2005; Rodríguez, 1995), lo que implicaba un trabajo competencial.

Por lo que respecta a la materia de Ciencias del deporte, y siguiendo los objetivos marcados por el primer nivel de concreción, estipulado por la Administración, se establecieron objetivos y contenidos para las diferentes unidades didácticas. De la multitud que se trabajaron, en la Tabla 1 se enumeran los principales relacionados con el proyecto (Tabla 2).

Tabla 2. Principales objetivos de aprendizaje, relacionados con los contenidos trabajados

\begin{tabular}{|c|c|c|}
\hline UD & Objetivos de aprendizaje & Contenidos \\
\hline 1 & $\begin{array}{l}\text { Analizar la significación del juego del } \\
\text { marro en la escuela con el fin de tener } \\
\text { una base para poder elaborar el torneo }{ }^{2}\end{array}$ & $\begin{array}{l}\text { - Descifrar el reglamento del juego } \\
\text { - Comprensión de la organización de los torneos } \\
\text { - Valoración de la dificultad en llevar a cabo un } \\
\text { proyecto autogestionado por el alumnado }\end{array}$ \\
\hline 2 & $\begin{array}{l}\text { Utilizar las pautas óptimas para } \\
\text { prevenir accidentes }\end{array}$ & $\begin{array}{l}\text { - Prevenciones a tener en cuenta para una buena } \\
\text { práctica deportiva, mediante diferentes medidas } \\
\text { de seguridad y de preparación para la actividad } \\
\text { - Análisis de las prevenciones a tener en cuenta } \\
\text { para la buena llevada a cabo del torneo }\end{array}$ \\
\hline 3 & $\begin{array}{l}\text { Diseñar y llevar a la práctica una } \\
\text { coreografía grupal, con el uso de la } \\
\text { música como elemento principal }\end{array}$ & $\begin{array}{l}\text { - Utilización óptima del ritmo con la música y los } \\
\text { pasos } \\
\text { - Cooperación y organización con los compañeros } \\
\text { para la creación de una coreografía }\end{array}$ \\
\hline 4 & $\begin{array}{l}\text { Organizar, dinamizar y controlar de un } \\
\text { torneo de marro para un grupo de } \\
\text { población específico y con fines } \\
\text { recreativos }\end{array}$ & $\begin{array}{l}\text { - Planificación de las tareas a realizarse para una } \\
\text { correcta organización del evento } \\
\text { - Ejecución de las intervenciones de control del } \\
\text { torneo de marro } \\
\text { - Motivación a los participantes y control de la } \\
\text { actitud durante el evento }\end{array}$ \\
\hline
\end{tabular}

\subsection{METODOLOGÍA}

La metodología utilizada en este proceso educativo fue la de investigación-acción (Ander-egg, 1990; Carr y Kemmis, 1988; Elliott, 2002; Kemmis, 1989; Kolb, 1984; Latorre, 2003; Lewin, 1946; Santiago, 1991; Woods, 1987). La justificación viene dada por nuestra propuesta de raíces dewenianas centrada en el método de proyectos y que es a la vez un elemento que se relaciona con este tipo de trabajos basados en la cesión de autonomía al grupo (Hipkin, 1977; Kolb, 1984; Lewin, 1946).

El artículo de Brasó y Torrebadella (2014) puede servir de guía de este objetivo. 
A la vez, el propio proceso - democrático, activo, significativo, con un papel secundario del profesor, etc. - se relaciona con los ideales de la pedagogía crítica y su enfoque de la enseñanza y aprendizaje mediante el currículo experimental (Apple, 1986; Gimeno, 2013; Giroux, 1990; Latorre, 2003; Mclaren, 1995). Esta metodología activa, práctica, no rutinaria y no autoritaria ayuda a proveer experiencias de éxito en los aprendizajes del alumnado (Blanco, 1906; Hipkin, 1977).

En el caso que nos ocupa, se secuenció el proceso en diferentes etapas. Primeramente, se elaboró en una fase preparatoria el plan de acción, concretado en un marco teóricoconceptual y en la planificación de actividades. Posteriormente, se pasó a la fase de actuación o trabajo de campo, materializado en las reflexiones diarias del alumnado, juntamente con sus aportaciones en las tareas de diversa índole — prescriptivas y voluntarias, individuales y en grupo- - El tratamiento de todos estos datos, en una fase analítica, permitió reflexionar en una fase final informativa sobre los resultados de la investigación-acción (Latorre, 2003; Rodríguez, Gil y García, 1996) detallados en el apartado final de conclusiones.

Como consecuencia, este procedimiento se caracterizó en diferentes aspectos remarcables. Así, los diversos modos de agrupamiento, la autonomía por parte de los propios discentes en la elaboración de actividades, la variedad de herramientas utilizadas, o el tratamiento global de los contenidos fueron aspectos significativos y esenciales que iban acorde con la metodología de investigación-acción.

Por lo que respecta a las diferentes agrupaciones, se crearon inicialmente equipos de trabajo entre los cuales estaba el grupo coordinador. Las otras tareas se repartieron y se iban exponiendo resultados al grupo de seguimiento y control y, posteriormente, al profesor. Esta fue la estructura principal del curso, aunque no fue la única. Así, durante todo el proceso la organización fue también en parejas, normalmente para tratar tareas voluntarias, terminar de perfeccionar el reglamento o elaborar un dossier de jugadas estratégicas del juego. También hubo otras agrupaciones como las que se hicieron para elaborar las coreografías que posteriormente se exhibirían el día del evento. Cabe destacar que también hubo un espacio para las actividades individuales, normalmente llevadas a término por propia iniciativa de cada alumno y con carácter voluntario. De este modo hubo diferentes formas de participación que permitieron a los estudiantes demostrar su esfuerzo y calidad de sus propuestas.

En relación con la autonomía cedida al alumnado, esta también fue variable. Inicialmente, el profesor, mediante sesiones directivas, detalló las características del juego que se iba a tratar, el que además era desconocido por la mayoría de alumnos; asimismo se hicieron unas sesiones prácticas. Posteriormente, en los inicios de la organización del evento, el docente estipuló algunas de las actividades mínimas que se tenían que llevar a cabo, estableciendo también la fecha de entrega. Con el paso del tiempo, los alumnos se fueron organizando más y mejor y la responsabilidad y tutela fue desplazándose al grupo clase. Este proceso fue muy positivo en la medida que mientras se avanzaba en el proyecto los alumnos se iban organizando solos, controlados por el grupo coordinador y con la figura del profesor desplazada a un segundo término. El mismo grupo organizador llegó a ser el canalizador de la evaluación en relación con las entregas y trabajos a presentar por parte de los diferentes grupos, ya que todas las actividades, antes de llegar al profesor, pasaban por este grupo.

Las herramientas utilizadas fueron establecidas en gran medida sobre las necesidades para el logro del evento. En relación con las tecnologías — un campo, por cierto de mucha importancia en los tiempos actuales_-, son destacables los diferentes videos que se hicieron para la promoción y recuerdo del torneo. A la vez, las presentaciones fotográficas en formato 
digital y los programas de diseño para la elaboración del logotipo fueron esenciales. Otras herramientas utilizadas fueron las relacionadas con la elaboración de pancartas o las referentes a aspectos de tratamiento de la voz y la música para el día del evento.

\subsection{ACTIVIDADES DEL PROYECTO Y TRANSVERSALIDAD CON OTRAS MATERIAS}

Para comprender el funcionamiento de un juego, y por lo tanto su la lógica interna, hay que practicarlo. Por lo tanto, la actividad inicial del proyecto fue la práctica del propio juego. Estas primeras partidas, que contemplaron una breve explicación histórica y el objetivo del proyecto, despertaron el interés de los alumnos y este fue el punto de inicio del proyecto. A partir de aquí los participantes se agruparon según sus afinidades y empezó el trabajo autónomo de los diferentes equipos.

Un factor inicial que había que concretar era el reglamento. Como consecuencia, el grupo organizador - después de una revisión de las variantes encontradas- estableció diferentes partidas con variedad de normativas y espacios a criterio del grupo encargado de detallar el reglamento, hecho que permitió posteriormente fijar las condiciones óptimas para el día del torneo. Es destacable decir que la problemática del juego radica en la dificultad del arbitraje, a causa de la complejidad de la lógica interna (Brasó y Torrebadella, 2014; Torrebadella y Brasó, 2014). Por lo tanto, un reglamento simple y claro era indispensable para el buen funcionamiento el día del encuentro. Posterior a estas sesiones iniciales, la elaboración del vídeo promocional, la preparación de las exposiciones informativas del torneo y la elaboración de trípticos e inscripciones fueron actividades que iban concretándose paulatinamente. También se trataron aspectos relacionados con la vestimenta y la mejor opción de enfrentamiento entre los equipos. En relación a este último aspecto y teniendo en cuenta que había ocho equipos, se optó por hacer dos 'liguillas' de cuatro equipos, que enfrentaría a cada campeón de su respectivo grupo a un partido de $25^{\prime}$. Los primeros de cada grupo, al final del torneo, realizarían un último partido que determinaría el ganador.

Se debe decir que se hicieron también preparativos para dar al evento mayor emoción y calidad. Por este motivo se elaboraron coreografías, a modo de inauguración y clausura. Además, se trataron aspectos relacionados con las informaciones que habría que dar, las tareas a realizar y otras cuestiones secundarias como la preparación del botiquín de primeros auxilios o la delimitación de zonas de acceso prohibido. Así, inicialmente se trataron aspectos motrices del juego y, posteriormente los aspectos relacionados con la preparación del torneo.

Por lo que respecta a los contenidos transversales e interdisciplinares del proyecto, estos fueron un aspecto más que visible. Numerosas actividades favorecían el trabajo competencial (Tabla 3). De este modo, había relación con las materias de Lengua en la medida que los alumnos tenían que hacer redacciones del reglamento, elaborar actas, dar noticias del evento, además de trabajar la parte oral mediante la exposición a los compañeros sobre aspectos relacionados con temas organizativos, y también a través de las exposiciones que se hacían a cada clase de participantes, detallando la organización y reglamentación del evento. Juntamente con estos aspectos, la elaboración de un diccionario de conceptos fue una tarea también relacionada con el ámbito lingüístico.

También la propia elaboración del reglamento para que el juego fuese entendido y de la manera más sencilla posible, desencadenó en la regularización del campo de juego. Por lo tanto, el cálculo de distancias de los límites del campo y de las zonas principales, significó un trabajo relacionado con la materia de Matemáticas. 
Asimismo, en el ámbito visual y de la plástica, además de los aspectos correspondientes a las proporciones del terreno y al dibujo técnico, tuvo importancia la elaboración de carteles, anuncios, trípticos y camisetas.

Respecto al ámbito de la música, hubo una relación directa mediante la expresión corporal. La elaboración de coreografías que se tendrían que llevar a término en el torneo, hizo que los alumnos tuviesen que tratar el tema en cuestión. Además, la búsqueda de canciones para el evento fue otro elemento relacionado con la música.

También se trataron temas históricos mediante la búsqueda de reglamentos antiguos o fotografías del evento a inicios del siglo XX. Así, esta búsqueda histórica fue clave para normativizar y promocionar el torneo.

Tabla 3. Ejemplo de actividades que se llevaron a cabo, agrupadas en las diferentes áreas

\begin{tabular}{|l|l|}
\hline Área & \multicolumn{1}{c|}{ Ejemplo de actividades } \\
\hline \multirow{4}{*}{ Área de lengua } & $\begin{array}{l}\text { - Elaboración del reglamento } \\
\text { - Creación de un diccionario de conceptos } \\
\text { - Exposiciones orales a compañeros y a los grupos participantes, relacio- } \\
\text { nadas con el torneo } \\
\text { - Redacciones y comentarios escritos del evento } \\
\text { - Artículos en formato de noticia }\end{array}$ \\
\hline Área de matemáticas & $\begin{array}{l}\text { - Estipulación de medidas y zonas del campo } \\
\text { - Llevada a cabo de la puntuación y clasificaciones del torneo }\end{array}$ \\
\hline \multirow{2}{*}{$\begin{array}{l}\text { Área de visual, } \\
\text { plástica y tecnología }\end{array}$} & $\begin{array}{l}\text { - Dibujo del campo de juego } \\
\text { - Creación de carteles, panfletos, trípticos y anuncios [figura 1 y 2] }\end{array}$ \\
\hline $\begin{array}{l}\text { - Elevada a cabo de fotografías y filmaciones } \\
\text { - Creación de camisetas para el grupo organizador } \\
\text { - Elaboración del póster fotográfico final, presentaciones del vídeo promo- } \\
\text { cional y final del evento, etc. }\end{array}$ \\
\hline Área de música & $\begin{array}{l}\text { - Escogida de música óptima relacionada con el torneo } \\
\text { - Coordinación de los pasos coreográficos con la música }\end{array}$ \\
\hline Área de historia & $\begin{array}{l}\text { - Búsqueda de material relacionado con el juego del marro a inicios del } \\
\text { siglo XX: fotos, reglamento, organización }\end{array}$ \\
\hline $\begin{array}{l}\text { Área de gestión, } \\
\text { animación, dinámica }\end{array}$ & $\begin{array}{l}\text { - Organización del torneo: secuenciación de actividades, relaciones inter- } \\
\text { personales, recursos humanos, recursos materiales }\end{array}$ \\
\hline
\end{tabular}

Consultable en Pereira et al. (2014).

$4 \quad$ Una muestra más del carácter global del proyecto es la aparición de la noticia en un periódico digital, a partir del redactado de una alumna. Disponible en: < http://titularscat.blogspot.com.es/2014/04/el-car-de-sant-cugat-recupera-el.html> Acceso el 11/10/2014.

5 Estas producciones permitieron la edición de un cortometraje del evento, por parte del grupo GR5, de Sabadell (tomado el 7 de enero de 2015): http://youtu.be/A5oAnmK5jHY

${ }^{6} \quad$ Uno de los vídeos promocionales del evento se puede consultar en Brasó (2014). 
Finalmente, todo el aspecto organizativo y de gestión fue otro elemento transversal a tratar. Los alumnos gestionaron todo el torneo, de inicio a fin, hecho que permitió implicar a los participantes en su propio proceso de enseñanza y aprendizaje $\mathrm{y}$, como consecuencia, incrementar su motivación (González, 2003; Lorente, 2004, 2008; Lorente y Joven, 2009). De este modo, tuvieron que tener presente toda la secuenciación de actividades que habría, organizar el material necesario, detallar los recursos humanos necesarios, organizar los equipos o dar informaciones a los participantes.

Figura 1. Póster del evento

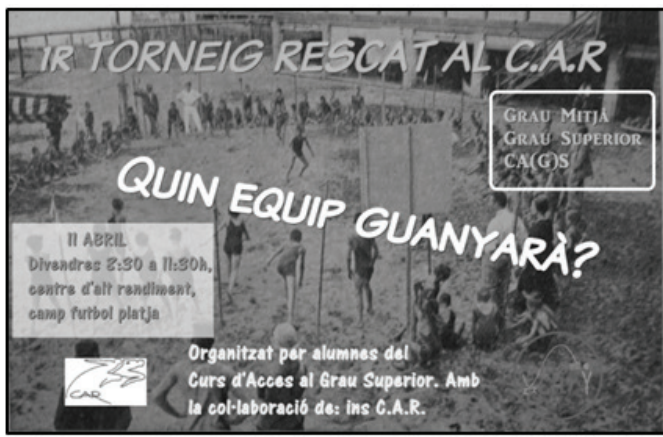

Cartel con fotografia de una partida de marro entorno al año 1930 en la Escuela del Mar

Figura 2. Póster del evento

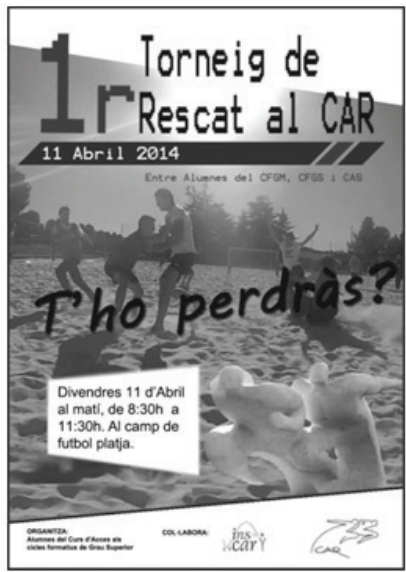

Cartel con fotografía de una partida de marro

llevada a cabo por los propios organizadores 
Figura 3. Acta del partido elaborada por el grupo correspondiente

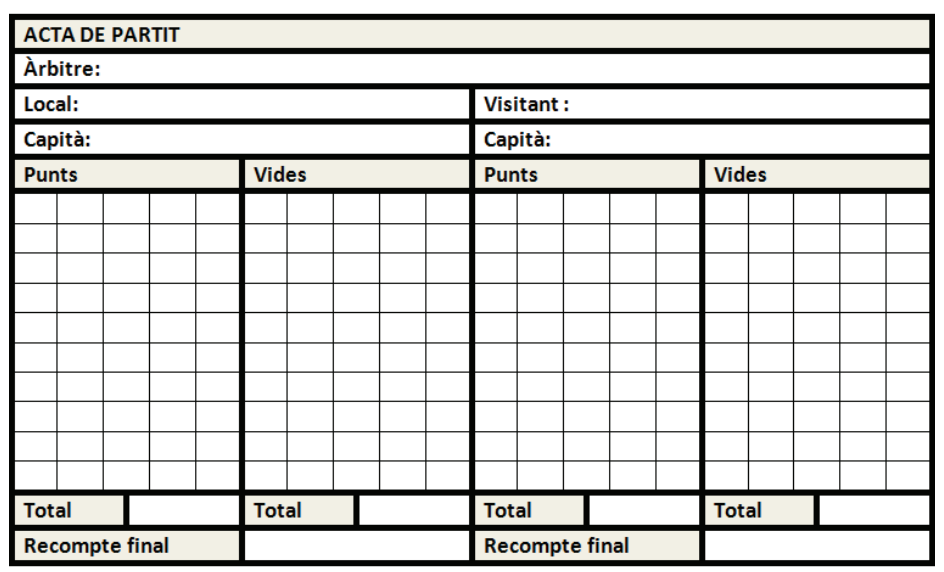

El recompte final serà les la resta del total de vides per el total de punts de l'equip contrari. (Exemple: si el local fa 5 punts i el visitant fa 3 vides el resultat serà de 2 punts)

\subsection{EVALUACIÓN}

El valor calificativo del proyecto fue en torno al $25 \%$ de la nota global de la materia. Este hecho se justifica si se tiene en cuenta que la duración del conjunto de actividades y la aparición de contenidos referentes al juego objeto del proyecto se trabajó en cuatro de las cinco unidades didácticas.

La evaluación de los alumnos y su trabajo en grupo en el proyecto se analizó de manera continua. Así, desde inicio de curso hasta el día del evento, se fueron tratando los diferentes temas para organizar la diada con éxito.

Para objetivar la evaluación se elaboró una tabla con diferentes ítems para poder analizar las competencias profesionales hacia la responsabilidad en el trabajo, la iniciativa personal, la autonomía o la relación interpersonal, entre otros aspectos. Los elementos que se trataron fueron varios:

a) Las actividades y tareas diarias, es decir, relacionadas con la actitud en las sesiones, aportación de ideas durante las clases o la eficiencia en las propuestas durante el curso, entre otros muchos aspectos.

b) Las tareas obligatorias y el grado de complejidad según el repartimiento de trabajos y gestiones a realizar, y canalizadas por el grupo coordinador o por el mismo profesor. Estas actividades fueron el establecimiento del reglamento, la elaboración de las actas, el diseño de los trípticos, la realización del video promocional, el análisis del modo de torneo más idóneo.

c) Las tareas voluntarias y el grado de complejidad, que consistían en trabajos como la elaboración de un logo para estampar en las camisetas, la elaboración de un álbum de fotos posterior al torneo, la puesta a punto del material informativo, la revisión del material óptimo para que el evento se pudiese realizar.

d) La implicación y gestión de las actividades del día del evento, según las responsabilidades preestablecidas capitaneadas por el grupo control. De este modo había tareas de arbitraje durante el encuentro (Figura 4), de apuntadores, de informadores, de 
megafonía, de repartimiento de premios (Figura 5), de periodista o de servicio de enfermería.

Es destacable la autonomía concedida al alumnado en sus agrupaciones, así como en tratar y llevar a cabo las tareas por las cuales estaban motivados.

Juntamente con estos aspectos, las entrevistas individuales en las que se reflexiona sobre el proceso, así como la encuesta final completada por los discentes fue un elemento más evaluativo. Somos conscientes de la falta en algunos momentos de evaluación formativa y compartida (López, 2006; López et al., 2007), justificable en parte por la importancia de la nota académica, que era un elemento decisorio para poder acceder al ciclo de grado superior. De todas formas, la valoración positiva del evento por parte de alumnos, profesores y del Instituto tiene que permitir una reflexión para nuevas propuestas y promover aun más modelos auto-educativos, con evaluaciones centradas en el proceso de enseñanza y con implicación del propio alumnado (López et al., 2012; Martos et al., 2014). Por lo tanto, habría que proponer procesos evaluativos más democráticos y justos.

Figura 4. Torneo de marro en el CAR

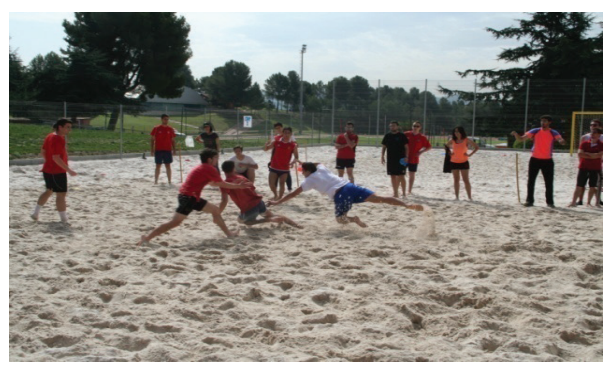

Figura 5. Torneo de marro en el CAR

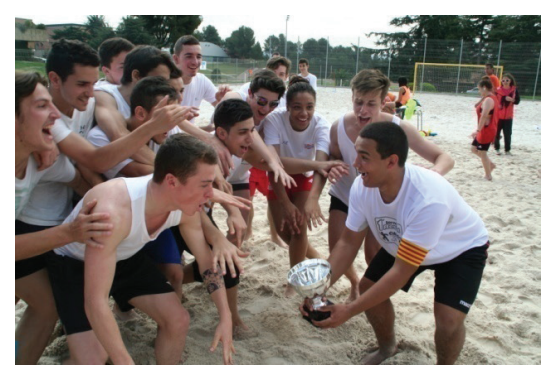

Momento de la final del Primer torneo de marro, 2014. El equipo ganador, en el momento de levantar el trofeo.

Por lo que respecta a la evaluación del proceso de enseñanza y aprendizaje, las reflexiones, consideraciones y problemáticas que surgían fueron de mucha validez para corregir diferentes enfoques de la propuesta, principalmente relacionadas con aspectos organizativos, temporales y también sociales. Para cumplimentar este último aspecto, se aplicó un cuestionario de valoración del proyecto. Este hecho permitió concluir que la cesión de la responsabilidad del tutor incrementó la motivación de los alumnos, tal y como puntualizó la mayoría del grupo. Además, la globalidad de aspectos que significaba la organización del proyecto: trabajo en grupo, adecuación al tiempo, liderazgo de un jefe, fue otro aspecto destacado como positivo, así lo hicieron notar los participantes, y transferible para su futuro, tanto formativo como laboral.

\section{A MODO DE CONCLUSIÓN}

En resumen y a la luz de los resultados obtenidos, nuestra experiencia ha mostrado que un aumento en la autonomía del alumnado en la elección de las tareas compartidas en grupo a realizar durante el desarrollo de las clases de educación física incrementa la satisfacción 
de una de las necesidades básicas como es la autonomía, lo que conlleva un aumento en la motivación, autodeterminada a nivel situacional y contextual. De este modo, se valora como positivo el papel del docente en el sentido de estructurar las sesiones cediendo parte de la autoridad a los protagonistas del proyecto educativo.

Las investigaciones futuras deberán seguir profundizando, desde posiciones experimentales o longitudinales siempre que sea posible, en los mecanismos sociales que determinan la satisfacción de las distintas necesidades básicas, a la vez que se analicen las relaciones con las diferentes consecuencias motivacionales y también entre los diferentes niveles de interés. Igualmente, se hacen necesarios más trabajos que estudien diferentes formas de estructurar las clases de educación física, y también en el ámbito deportivo, para comprobar los distintos efectos de satisfacción de estas estructuras, ello permitirá, en definitiva, determinar las mejores formas de incrementar la participación hacia la práctica de actividades físicas y deportivas.

La motivación constante y creciente de docentes y discentes es otro aspecto a valorar positivamente. Dos elementos estimuladores fueron el progresivo self-government que se dio al grupo, juntamente con la transversalidad de la propuesta. Estos hechos hicieron que el alumnado participase según sus propios intereses, ya que en la medida de lo posible se podían escoger tareas de una tipología acorde con las inquietudes personales.

Somos conscientes de la subjetividad evaluativa en la medida que cada alumno trabajaba en un tema distinto, y por lo tanto había tareas más laboriosas que otras. De todas formas, este aspecto se solucionó proponiendo tareas voluntarias, como por ejemplo elaborando un glosario de conceptos o un conjunto de estrategias para el juego. De este modo, el grupo o el alumnado con tareas de menos implicación podía optar por trabajar y colaborar más. Como consecuencia, estas actividades sirvieron para mejorar la objetividad en la tarea evaluativa.

Teniendo en cuenta la amplitud de la mayoría de currículos en el área de la educación física, el proyecto puede ser transferible tanto en la materia de educación física en diferentes cursos, como enmarcado en trabajos de síntesis o investigación. Una de las principales adaptaciones a tener en cuenta es la cesión de autonomía de alumnado según la edad y su nivel madurativo. Es remarcable también destacar que las conductas proactivas y prosociales del alumnado hicieron perder sentido subjetivo a la evaluación, y más teniendo en cuenta el éxito alcanzado por la organización del grupo en el torneo.

Finalmente, es remarcable apuntar que la idea inicial del sistema por proyectos traslada la propia idea del proyecto al alumnado. Como se ha visto, no se ha dado esta libertad inicial al alumnado, ya que se le ha impuesto la propuesta. Pero a diferencia de la mayoría de estos proyectos, éste ha sido mucho más complejo en la medida que el fin no era estático, como elaborar una construcción, un juguete, aprender una habilidad o diseñar una exposición, sino que era dinámico, sin saber, hasta el final, si había sido satisfactorio y exitoso.

En definitiva, el objetivo de la experiencia buscaba ceder la responsabilidad al alumnado en su proceso de aprendizaje. Se busca así que en este modelo auto-educativo el sujeto se transformase en "un gran educador en sí mismo" (Blanco, 1906: 174).

\section{REFERENCIAS BIBLIOGRÁFICAS}

Almeida, A. S. (2003). Les "Public Schools" i la reforma educativa de Thomas Arnold (1828-1842).

Temps d'Educació, 27, 305-329. 
Ander-Egg, E. (1990). Repensando la investigación-acción participativa. Buenos Aires: Lumen Humanitas.

Angulo, F., Betanzo, M. J., \& López, M. M. (2005). Viviendo los contenidos: una experiencia en el uso de tecnologías móviles en los procesos de Enseñanza y Aprendizaje. Quadernsdigitals.net., 37. Recuperado desde: http://www.quadernsdigitals.net/index.php?accionMenu=hemeroteca. VisualizaArticuloIU.visualiza\&articulo_id=8419

Apple, M. (1986). Ideología y currículo. Madrid: Akal.

Bantulà, J. (2006). La introducció dels jocs populars i tradicionals en l'educació escolar dels infants. Temps d'Educació, 8, 235-247.

Barbero, J. I. (1996). Cultura profesional y currículum (oculto) en educación física. Reflexiones sobre las (im) posibilidades del cambio. Revista de Educación, 311, 13-49.

Becerro, A., \& Torrebadella, X. (2013). El joc tradicional i popular a l'escola primària: una proposta de l'educació física cap a la cessió d'autonomia. REIRE:Revista d'Innovació i Recerca en Educació, 6(2), 100-118.

Belando, N., Ferriz-Morell, R. F., \& Moreno-Murcia, J. A. (2012). Mejora personal y social a través de la promoción de la responsabilidad en la actividad físico-deportiva. RICYDE. Revista Internacional de Ciencias del Deporte, 8(29), 202-222.

Blanco, R. (1906). Tratado de pedagogía. Madrid: Revista de Archivos.

Blanco, R. (1996). Un clásico del siglo XX: J. Dewey. Revista de Educación, 311, 397-407.

Brasó, J. (2014). L'anunci publicitari d'un projecte educatiu: Organització d'un torneig del joc popular del rescat o marro. Lecturas: Educación Física y Deportes, 19(196). Recueprado desde: http://www.efdeportes.com/efd196/torneig-del-joc-popular-del-rescat-o-marro.htm

Brasó, J., \& Torrebadella, X. (2014). El joc del rescat a Catalunya. Un projecte educatiu a l'Escola del Mar de Pere Vergés. Temps d'Educació, 47, 191-212. Recueprado desde: http://www. publicacions.ub.edu/revistes/tempsdeducacio47/default.asp?articulo=1031\&modo=abstract $>$

Butts, M. (1930). Los centros de interés, los ‘proyectos' y la simplificación del empleo del tiempo. En VVAA, Cómo debemos educar a nuestros hijos (pp. 21-25). Barcelona: Bauzá.

Camerino, O., \& Castañer, M. (1984). Educació física escolar: jocs i esports populars al Pallars. Barcelona: Generalitat de Catalunya-Institut Nacional d'Educació Física.

Carr, W., \& Kemmis, S. (1988). Teoría crítica de la enseñanza. España: Martínez Roca.

Cecchini, J. A., Montero, J., \& Peña V. (2003). Repercusiones del programa de intervención para desarrollar la responsabilidad personal y social sobre los comportamientos de fair-play y el autocontrol. Psicothema, 15, 631-637.

Dahlman, L. M. (2011). El discurso pedagógico en la obra de Fray Martín Sarmiento (1695-1772). Trocadero, 1(10-11), 7-48.

De Borja, M. (1984). El juego como actividad educativa: Instruir deleitando. Barcelona: Ediciones de la Universitat de Barcelona.

Devís, J. (2008). El juego en el currículum de la Educación Física: aproximación crítica (o el reino de lo posible en la postmodernidad). Revista de Educación Física y Deporte, 27(2), 79-89.

Devís, J. \& Peiró, C. (1992). Nuevas perspectivas curriculares en educación física: la salud y los juegos modificados. Barcelona: INDE.

Elliott, J. (2002). La investigación acción. Madrid: Morata.

Ferrándiz, C. (2005). Evaluación y desarrollo de la competencia cognitiva. Madrid: Ministerio de Educación y Ciencia. Secretaría General Técnica.

Gimeno, P. (2013). Reflexiones críticas y autocríticas sobre Pedagogía Crítica. Revista Interuniversitaria de Formación del Profesorado, 27(2), 77-92.

Giroux, H. A. (1990). Los profesores como intelectuales. Hacia una pedagogía crítica del aprendizaje. Barcelona: Paidós.

Gómez, I., \& Prat, M. (2009). Hacia una Educación Física crítica y transformadora de las prácticas sociales: de la práctica a la reflexión y viceversa. Cultura y Educación, 21(1), 9-17. 
González-Agàpito, J. (1998). Pere Vergés i Farrés (1896-1970). Barcelona: Ajuntament de Barcelona.

González, C. (2003). Un modelo de autogestión de las actividades extraescolares en un centro de educación secundaria. Apunts: Educación física y deportes, 74, 24-33.

GutsMuths, J. (1796). Spiele zur übung und erholung des körpers und geistes, fur die jugend. Schnpfenthal: ImVerlage der Buchhandlung der Erziehungsanstalt.

Hipkin, J. (1977). Aprendizaje en grupos. En P. Buckman (Dir.), Educación sin escuela (pp. 111-121) Barcelona: Península.

Kemmis, S. (1989). Investigación en la acción. En T. Husen \& T.N. Postlethwaite, Enciclopedia Internacional de la Educación (pp. 3330-3337). Barcelona: Vicens Vives-M.E.C.

Kirk, D. (1990). Educación física y currículum. Valencia: Universitat de Valencia.

Kolb, D. (1984). Experiential learning. Experience as The Source of Learning and Development. New Jersey: Prentice-Hall.

Lacueva, A., Imbernón, F., \& Llobera, R. (2004). Los peces: una unidad didáctica en una escuela "diferente". RELIEVE, 10(2), 211-244.

Latorre, A. (2003). Conocer y cambiar la práctica educativa. Barcelona: Graó.

Lavega, P. (2006). El juego y la tradición en la educación de valores. Educación Social, 33, 54-72.

Lewin, K. (1946). Action Research and Minority Problems. Journal of Social lssues, 2(4), 34-46.

López, A. M., \& Lacueva, A. (2007). Proyectos en el aula: Cinco categorías en el análisis de un caso. REICE: Revista Electrónica Iberoamericana sobre Calidad, Eficacia y Cambio en Educación, 5(1), 78-120.

López, V. (Coord.). (2006). La evaluación en educación física. Revisión de modelos tradicionales y planteamiento de una alternativa. La evaluación formativa y compartida. RETOS. Nuevas tendencias en educación física, deporte y recreación, 9(10), 31-41.

López, V. (2012). Didáctica de la educación física, desigualdad y transformación social. Estudios Pedagógicos, XXXVIII, Número Especial, 155-176.

López, V., \& Gea, J. (2010). Innovación, discurso y racionalidad en Educación Física. Revisión y prospectiva. Revista Internacional De Medicina y Ciencias De La Actividad Física y Del Deporte, 10(38), 245-270.

López, V.M., Barba, J.J., Monjas, R., Manrique, J.C., Heras, C., González, M., \& Gómez, J.M. (2007). Trece años de evaluación compartida en Educación Física. Revista Internacional de Medicina y Ciencias de la Actividad Física y el Deporte, 7(26), 69-86.

López, V.M., Kirk, D., Lorente, E., MacPhail, A., \& Macdonald, D. (2012). Alternative assessment in physical education: a review of international literature. Sport, Education and Society, 18(1), 57-76.

Lorente, E. (2004). La autogestión en educación física. Un estudio de caso en Secundaria (Tesis doctoral). Institut Nacional d'Educació Física (UB). Departament de Teoria i Història de l'Educació, Barcelona.

Lorente, E. (2008). Estimular la responsabilidad y la iniciativa: Autogestión en Educación Física. Apunts. Educación física y deportes, 92, 26-34.

Lorente, E., \& Joven, A. (2009). Autogestión en Educación Física: una investigación etnográfica. Cultura y Educación, 21(1), 67-79.

Martín, X. (2011). Treball per projectes amb servei a la comunitat. Temps d'Educació, 41, 57-67.

Martos, D., Torrent, G., Durbà, V., Saiz, L., \& Tamarit, E. (2014). El desarrollo de la autonomía y la responsabilidad en educación física: Un estudio de caso colaborativo en secundaria. RETOS. Nuevas Tendencias en Educación Física, Deporte y Recreación, Junio-Diciembre, 3-8.

McLaren, P. (1995). Pedagogía crítica y cultura depredadora: Políticas de oposición en la era postmoderna. Barcelona: Paidós.

Mérida, R., Barranco, B., Criado, E., Fernández, N., López, R. M., \& Pérez, I. (2011). Aprender investigando en la escuela y en la universidad. Una experiencia de investigación-acción a partir del Trabajo por proyectos. Investigación en la escuela, 73, 65-76. 
Moreno, J. A., \& Vera, J. A. (2008). Un estudio experimental de las diferencias por género en la percepción de competencia a partir de la cesión de responsabilidad en las clases de educación física. Revista iberoamericana de educación, 46(8), 1-10.

Moreno, J. A., Aracil, A., \& Reina, R. (2014). La cesión de responsabilidad en la evaluación: una estrategia adaptada al Espacio Europeo de Educación Superior. Educación XX1, 17(1), 181-200.

Moreno, J. A., Gómez, A., \& Cervelló, E. (2010). Un estudio del efecto de la cesión de autonomía en la motivación sobre las clases de educación física. Motricidad: revista de ciencias de la actividad física y del deporte, 24, 15-27.

Moro, M. (2007). Quintiliano de Calahorra: didáctica y estrategias educativas en la antigua Roma. Foro de educación, 5(9), 125-132.

Navarro, V. (2011). Aplicaciones pedagógicas del diseño de juegos motores de reglas en educación física. Ágora para la educación física y el deporte, 1(13), 15-34.

Parlebas, P. (2001). Juegos, deporte y sociedades. Léxico de praxeología motriz. Barcelona: Paidotribo.

Pereira, J., Capó, G., Torrebadella, X., \& Brasó, J. (2014). Reglament del joc del rescat, riscat o marro. Lecturas: Educación Física y Deportes, 19(199). Recuperado desde: http://efdeportes. com/efd199/reglament-del-joc-del-rescat-o-marro.htm

Pring, R. (1976). Knowledge and Schooling. Somerest: Open Books Publishing Ltd.

Rich, J. (2000). Los modelos anglosajones y la organización en Francia del proyecto de escuela. Revista de Educación, 323, 123-134.

Rodríguez, M. L. (Coord.). (1995). Educación para la carrera y diseño curricular. Teoría y práctica de programas de educación para el trabajo. Barcelona: Universitat de Barcelona.

Rodríguez, G., Gil, J., \& García, E. (1996). Metodología de la investigación cualitativa. Granada: Aljibe.

Saladrigas, R. (1973). L'Escola del Mar i la renovació pedagògica a Catalunya. Barcelona: Edicions 62.

Santiago, P. (1991). Investigación-acción y competencia docente. Revista Interuniversitaria de Formación del Profesorado, 10, 351-357.

Torrebadella, X. (2011). Vicente Naharro y los juegos corporales en la educación física española de la primera mitad del siglo XIX. Ágora para la Educación física, 13(2), 165-182.

Torrebadella, X. (2013). Hacia un modelo de actividades físico deportivas inclusivas en el medio natural. EmásF. Revista Digital de Educación Física, 23, 1-15.

Torrebadella, X., \& Brasó, J. (2014). El juego del marro. Análisis de la lógica interna y posibilidades educativas. Tándem. Didáctica de la Educación Física, 45, 75-79.

Torres, J. (1987). La globalización como forma de organización del currículo. Revista de Educación, $282,103-130$.

Trobajo, N., \& Salvatella, P. (2003). El mètode de projectes. Revista de Girona, 216, 64-69.

Valserra, F. (1944). Historia del deporte. Barcelona: Ed. Plus-Ultra.

Varela, J. (1991). El cuerpo de la infancia. Elementos para una genealogía de la ortopedia pedagógica. En VVAA, Sociedad Cultura y Educación. Homenaje a la memoria de Carlos Lerena Alesón (pp. 229-248). Madrid: Universidad Complutense de Madrid.

Vera, J. A. (2010). Dilemas en la negociación del currículum con el alumnado a partir de la cesión de responsabilidad de la evaluación en el aula de educación física. Revista de Investigación en Educación, 7, 72-82.

Vera, J. A., \& Moreno, J. A. (2007). El pensamiento del profesorado en Educación Física ante la cesión de responsabilidad en la evaluación del alumnado. Profesorado. Revista de currículum y formación del profesorado, 11(2), 1-15.

Vera, J. A., \& Moreno, J. A. (2009). ¿Debemos ceder responsabilidad a los alumnos para que participen en la evaluación en educación física. Tándem. Didáctica de la Educación Física, 29, 91-96. 
Vergés, P. (1932). La Vida Espiritual a Vilamar. Conferència donada per... Barcelona: Ajuntament de Barcelona.

Vergés, P. (1947). Libro de Evocaciones (1922-1947). Barcelona: Altés.

Vergés, P. (1957). La Escuela del Mar. Una escuela y una vocación. Barcelona: Escuela del Mar.

Vicente, M. (2012). El discurso técnico de la educación física o el techo de cristal: Bosquejo de un debate sobre el código disciplinar de la educación física y su precaria legitimidad. Estudios pedagógicos, XXXVIII, Número Especial, 89-109.

Vicente, M., \& Brozas, M. P. (1996). La disposició regulada dels cossos. Proposta d'un debat sobre la cultura física popular i els jocs tradicionals. Apunts. Educació Física i Esports, 48, 6-16.

Vilanou, C. (1998). La pedagogia de Pere Vergés: un vitalisme espiritualista ludicoestètic. En J. González-Agàpito (Coord.), Pere Vergés i Farrés (1896-1970) (pp. 79-101). Barcelona: Ajuntament de Barcelona.

Vilanou, C., \& De Bolòs, O. (2005). Joventut, esport i religió: el moviment Muscular Christianity. Educació i Història: Revista d'Història de l'Educació, 7, 63-92.

Woods, P. (1987). La escuela por dentro. Barcelona: Paidos / MEC.

Zabala, A. (1999). Enfoque globalizador y pensamiento complejo. Barcelona: Graó. 
